\title{
Risk of Introduction of Drug-Resistant Malaria in a Non-Endemic Country, Kuwait: A Real Threat?
}

\author{
Jamshaid lqbal $^{\mathrm{a}}$ Ali Sher ${ }^{\mathrm{b}}$ P.R. Hira ${ }^{\mathrm{a}}$ Abdul Aziz Al-Anezic \\ aDepartment of Microbiology, Faculty of Medicine, Kuwait University, ${ }^{\mathrm{b}}$ Malaria Laboratory, \\ Ministry of Health, and 'Infectious Diseases Hospital, Ministry of Health, Kuwait
}

\section{Key Words}

Plasmodium falciparum . Malaria .

Chloroquine $\cdot$ Mefloquine $\cdot$ Immigrants

\begin{abstract}
Objectives: This study investigates the prevalence of drug-resistant malaria infection in Kuwait in patients with malaria infection to the two most important and primary antimalarial drugs, chloroquine and mefloquine. Study Subjects and Methods: In vitro screening of malarial parasites to chloroquine and mefloquine was done during 1994-1996 in all those cases who presented with malaria-like symptoms and had a parasite density of $\geq 1,000$ asexual pure Plasmodium falciparum parasites per microlitre of blood and had not taken any antimalarial drugs during the last 3 weeks. Results: During 1987-1997 the total number of malaria cases detected
\end{abstract}

\begin{tabular}{ll}
\hline KARGER & ( 2000 S. Karger AG, Basel \\
Fax +4161306 1234 $34-7571 / 00 / 0092-0125 \$ 17.50 / 0$ \\
$\begin{array}{l}\text { E-Mail karger@karger.ch } \\
\text { www.karger.com }\end{array}$ & $\begin{array}{l}\text { Accessible online at: } \\
\text { www.karger.com/journals/mpp }\end{array}$
\end{tabular}

ranged between 650 and 1,350 each year. More than $75 \%$ of the cases had Plasmodium vivax infection. The majority of these cases $(>98 \%)$ were detected in individuals coming from various malaria-endemic areas to reside or work in Kuwait. Of the 575 cases tested for drug resistance 42 isolates (7.3\%) were resistant to chloroquine (MIC $>8 \mathrm{pmol}$ ) and 6 isolates $(1.04 \%)$ were resistant to mefloquine (MIC $>64 \mathrm{pmol}$ ). The $50 \%$ inhibitory concentrations were 2.7 and 4.8 pmol for chloroquine and mefloquine, respectively. All the 6 isolates that were resistant to mefloquine were also resistant to chloroquine. Chloroquine resistance was seen in patients from various malaria-endemic countries. Conclusion: To date no indigenous case of malaria has been detected in Kuwait, however, recent environmental and demographic changes in and around the State of Kuwait may threaten the present status of zero en-

Dr. Jamshaid Iqbal

Department of Microbiology, Faculty of Medicine

Kuwait University, PO Box 24923

Safat (Kuwait)

E-Mail iqbal@hsc.kuniv.edu.kw 
demicity. In this study we show that $7.3 \%$ of the malaria isolates tested were resistant to chloroquine and $1.04 \%$ isolates were resistant to mefloquine.

Copyright $@ 2000$ S. Karger AG, Basel

\section{Introduction}

The development of antimalarial drug resistance has had a tremendous impact on malaria control. Drug resistance has been implicated in the spread of malaria to new areas and re-emergence of malaria in areas in which the disease had been eradicated [1]. The spread of drug-resistant strains of Plasmodium falciparum has made the prophylaxis and treatment of the disease increasingly difficult, particularly with chloroquine [2-5].

The State of Kuwait is located to the northwest of the Arabian Gulf. To the north and north-west it shares the borders with the Republic of Iraq, to the south and south-west with the Kingdom of Saudi Arabia and to the east with the Arabian Gulf. Due to Kuwait's location in a desert geographic region, its climate is characterised by long dry hot summers $\left(40-50^{\circ} \mathrm{C}\right)$ and short warm winters. Due to the strategic location, it is considered a natural gateway to the Arabian Peninsula, which has given it a long-standing commercial importance leading to influx of a large number of expatriates from all over the world.

Low-grade malaria is detected in the Arabian Gulf countries of The United Arab Emirates, Saudi Arabia and Oman. However, the State of Kuwait is still sustaining the zero reporting of indigenous malaria, while imported malaria is quite prevalent. Anopheline breeding places are relatively limited and thus, no malaria transmission has been detected in Kuwait. However, Kuwait is vulnerable to malaria infection as it is exposed to countries where there is transmission of ma- laria [6-10], and because of frequent influx of multinational expatriate work force from countries with high malaria endemicity. According to the Communicable Disease Control Unit of Preventive Medicine, Ministry of Health, Kuwait, 876, 654, 811 and 746 cases of malaria were detected in Kuwait in 19941997, respectively, and about 15-20\% of the total cases were of falciparum malaria.

We report in this study the current status of malaria infection and incidence of drugresistant malaria in the State of Kuwait and analyse the threat of introduction of drugresistant $P$. falciparum malaria in Kuwait in relation to emerging new risk factors.

\section{Patients and Methods}

\section{Screening and Case Detection}

All new arrivals coming to Kuwait from malariaendemic areas and applying for the residence or work permit undergo a medical check-up and screening for the detection of malarial parasite by microscopy of $10 \%$ Giemsa-stained thick and thin blood films. The bulk of this work is carried out by the Malaria Laboratory, Medical Centre for Labour Examination, Ministry of Health. Other malaria cases including Kuwaiti nationals were detected at the Infectious Diseases Hospital and Mubarak Al-Kabeer Teaching Hospital. All slide-positive cases are treated with chloroquine or mefloquine. All the treated patients are referred to the respective area clinics of the patients for radical treatment over a period of 8 weeks.

In vitro screening for chloroquine and mefloquine was done in all those cases who had a parasite density of $\geq 1,000$ asexual pure $P$. falciparum parasites per microlitre of blood and had not taken any antimalarial drugs during the last 3 weeks. The data included in this study was collected during 1994-1996. Of the 2,037 malaria cases detected during the study period, a total of 575 P. falciparum isolates were successfully tested for in vitro sensitivity to chloroquine and mefloquine. The majority of the parasite isolates were from individuals coming from India, Sri Lanka, Bangladesh and Pakistan. Three of the $P$. falciparum isolates were from the Kuwaiti nationals who had recently returned from malaria-endemic countries. A detailed personal and travel history was taken from each patient to deter- 


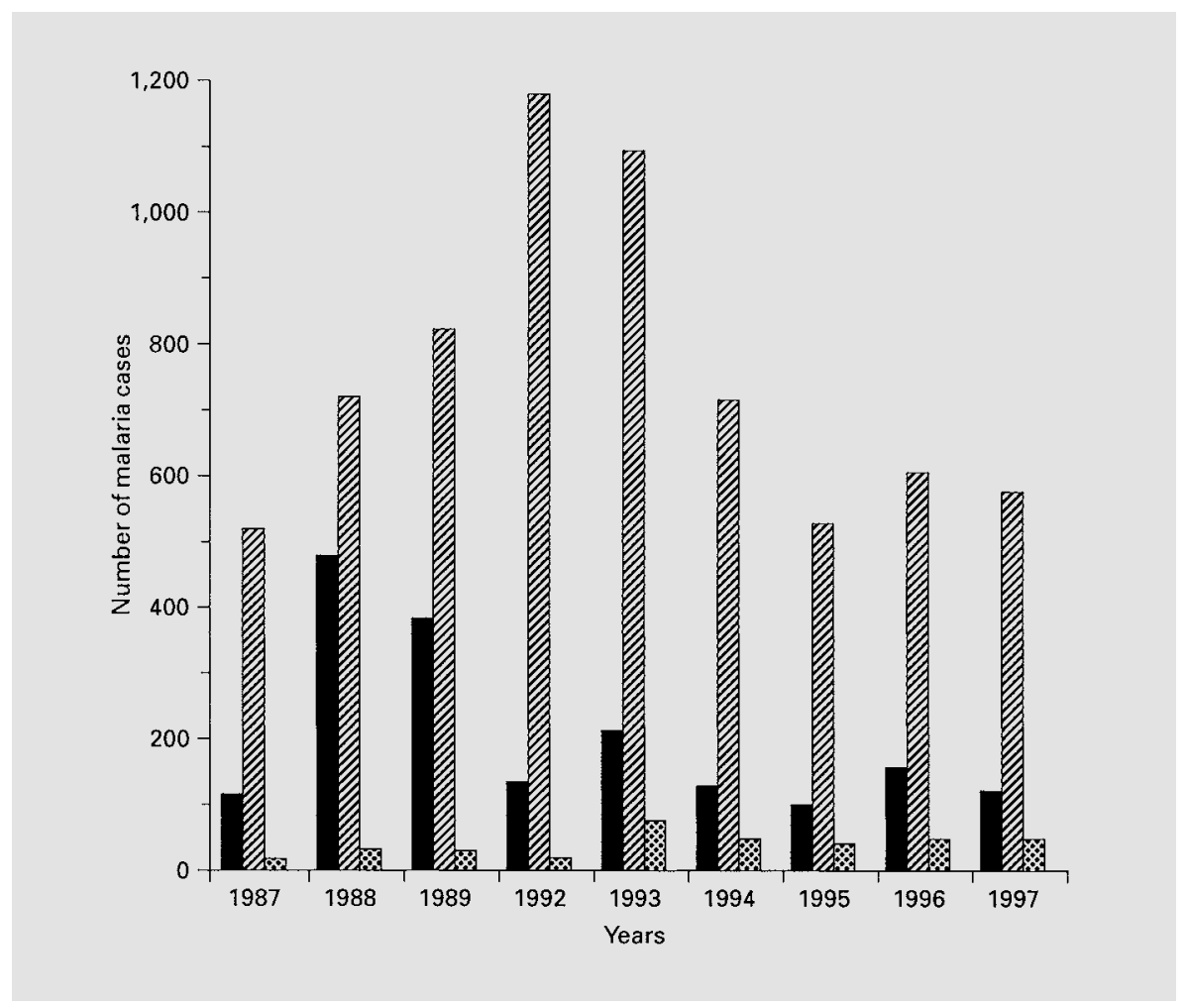

Fig. 1. Number of malaria cases detected in the immigrant workers by examination of Giemsa-stained thick and thin blood smears. During 1990-1991 the data was not available as the State of Kuwait was invaded and occupied by Iraq during this time. $\boldsymbol{\square}=P$. falciparum;

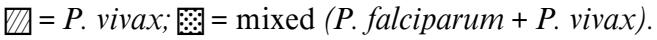

mine if the infection was acquired locally or imported.

\section{Screening for in vitro Chloroquine/Mefloquine} Resistance

The micro in vitro drug sensitivity tests were done on the predosed plates following manufacturer's instructions [11]. Briefly, $200 \mu \mathrm{l}$ of whole blood from each patient was collected by finger prick and added to $1.8 \mathrm{ml}$ of RPMI 1640 culture medium. Fifty microlitres of the diluted blood was added to each well coated with various concentrations of chloroquine or mefloquine. The plates were incubated at $37^{\circ} \mathrm{C}$ in $5 \%$ $\mathrm{CO}_{2}$ for $30 \mathrm{~h}$ and screened for mature schizonts on thick films. The assay was valid only if $\geq 10 \%$ of the parasites in the control well developed into schizonts with $\geq 3$ nuclei. The minimum inhibitory concentra- tion (MIC) was defined as the lowest drug concentration in which no such schizonts were observed. Concentrations are expressed as per well volume of bloodmedium mixture. The cut-off concentrations for in vitro resistance to the respective drugs were considered as $8 \mathrm{pmol}$ for chloroquine and $64 \mathrm{pmol}$ for mefloquine [11].

\section{Results and Discussion}

The incidence of slide-positive malaria cases, $P$. falciparum and Plasmodium vivax and mixed ( $P$. falciparum $+P$. vivax), during the years 1987-1997 is presented in figure 1 . 


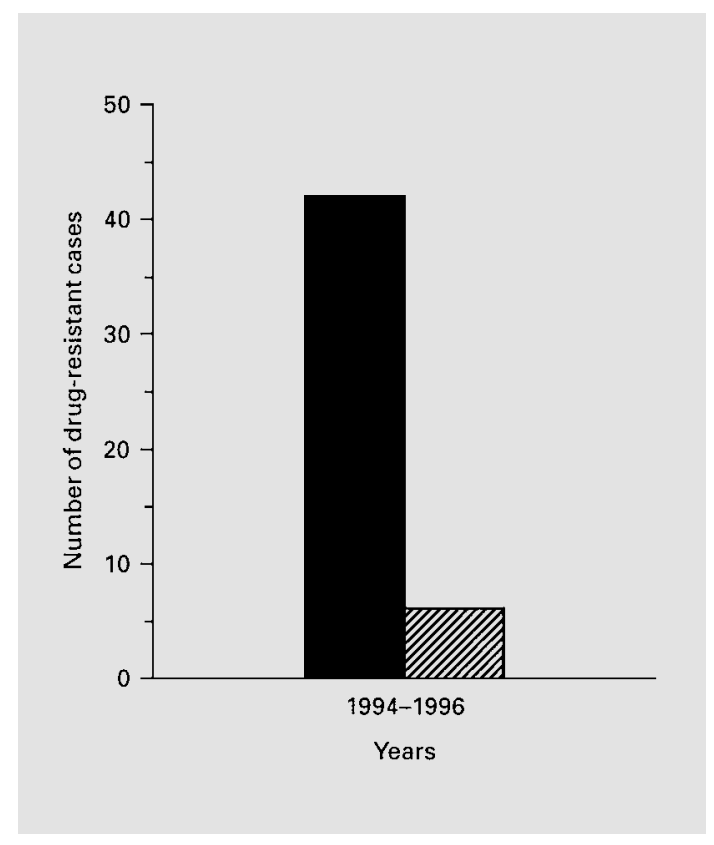

Fig. 2. The antimalarial drug susceptibility profile of 575 P. falciparum isolates from immigrant workers during 1994-1996. The in vitro drug susceptibility assays were done according to manufacturer's instructions [11] (chloroquine, mefloquine).

The total number of malaria cases detected each year ranged between 650 and 1,350, with $P$. vivax malaria being the predominant infection. The majority of these cases $(>98 \%)$ were detected in individuals coming from various malaria-endemic areas to reside or work in Kuwait. Less than $1 \%$ of cases were seen among the Kuwaiti nationals who had acquired the infection during their visit abroad. A total of 575 P. falciparum isolates were successfully tested for in vitro sensitivity to chloroquine and mefloquine. The majority of the malaria cases were from India, Sri Lanka, Bangladesh and Pakistan and only 3 isolates were from Kuwaiti patients who gave a history of travel to malaria-endemic areas (2 to India and 1 to Thailand).
It is now well documented that the movement of population plays an important role in the spread and introduction of malaria infection [6, 12-14]. Each year approximately 80,000 individuals come to work or reside in Kuwait from areas highly endemic for malaria and an average of 800 imported malaria cases are detected each year. The screening for malaria infection is mandatory for all those who come to Kuwait to work or reside from malaria-endemic areas. All the slide-positive malaria cases who present with fever are treated at the Infectious Diseases Hospital and are then followed up by the Preventive Health Department (area clinics) for the radical antimalarial treatment. The radical treatment includes chloroquine (300 $\mathrm{mg}$ base) and primaquine ( $30 \mathrm{mg}$ base) weekly for 8 weeks and is given to all malaria cases including falciparum malaria. This stringent regimen of radical therapy for falciparum malaria, though not recommended by the WHO, is implemented by the Preventive Department of the Ministry of Health to prevent the recrudescence and to eradicate the gametocytes, since in the majority of the malaria cases the parasitaemia is very low and the chances of missing some $P$. vivax in low numbers is high, especially in mixed infections. In addition, all the visiting students coming from malariaendemic countries and the workers at the water treatment plants and at the agricultural farms are screened for malaria each year irrespective of their visit abroad. Free consultation is given to the travellers seeking prophylactic measures prior to their trip to malariaendemic areas.

Due to Kuwait's location in a desert geographic location, anopheline breeding places are relatively limited and the present larval density levels of Anopheles stephensi and Anopheles pulcherrimus are well below the threshold for transmission. However, routine checks for vector breeding are carried out by ento- 
mologists who record mosquito larval counts at all field sites as well as in areas of residence of all the slide-positive cases.

The drug sensitivity data is shown in figure 2. Of the 575 cases tested 42 isolates $(7.3 \%)$ were resistant to chloroquine (MIC $>8$ pmol) and 6 isolates (1.04\%) were resistant to mefloquine (MIC >64 pmol). The 50\% inhibitory concentrations were 2.7 and 4.8 pmol for chloroquine and mefloquine, respectively. The corresponding 99\% concentrations were 31 and $81.2 \mathrm{pmol}$. All the 6 isolates that were resistant to mefloquine were also resistant to chloroquine. Chloroquine resistance was seen in patients from various malaria-endemic countries, however, mefloquine resistance was detected only in patients from the Indian subcontinent. Thus a wide distribution of chloroquine-resistance of $P$. falciparum in these malaria-endemic countries is directly influencing the malaria situation in Kuwait $[15,16]$.

Though no indigenous malaria case has so far been detected in the State of Kuwait, however, certain risk factors have emerged recently in and around Kuwait which may endanger the present situation of malaria in Kuwait. These factors are: (i) Reports of an increase of multidrug-resistant $P$. falciparum strains in countries from where the majority of the immigrant population comes from to work or reside in Kuwait [6]. (ii) A change in the present ecological and meterological conditions due to an enthusiastic drive of making Kuwait green and extending plantation throughout the whole country. Presently the larval density levels of $A$. stephensi and $A$. pulcherrimus are well below the threshold for transmission due to dry hot conditions which act as limiting factors against building up high population densities of $A$. stephensi. (iii) The extensive use of water in irrigation and for domestic purposes. (iv) Recent reports of malaria transmission and detection of chloro- quine-resistant cases in the indigenous population in the neighbouring States of Oman, United Arab Emirates and Saudi Arabia [6$10]$.

In conclusion, there is no local transmission of malaria infection in Kuwait. The majority of the malaria cases detected (98\%) were among the expatriate population coming from malaria-endemic countries. Less than $1 \%$ of malaria cases were seen among Kuwaiti nationals who had acquired the infection during their visit abroad. In this study we show that $7.3 \%$ of the malaria isolates tested in vitro for drug sensitivity were resistant to chloroquine and $1.04 \%$ isolates were resistant to mefloquine.

\section{Acknowledgements}

We thank the Ministry of Health, Kuwait, for providing us the technical information on the malaria control programme. The financial assistance for this project was provided by the Kuwait University Research Grant (MI 109). 


\section{References}

1 Bjorkman A, Phillips-Howard PA: The epidemiology of drug-resistant malaria. Trans R Soc Trop Med Hyg 1990;84:177-180.

2 Black RH, Bruce-Chwatt LJ: Chemotherapy of Malaria. Geneva, WHO, 1986.

3 Peters W: Chemotherapy and Drug Resistance in Malaria. London, Academic Press, 1987

4 Bickii J, Basco LK, Ringwald P: Assessment of three in vitro tests and in vivo test for chloroquine resistance in $P$. falciparum clinical isolates. J Clin Microbiol 1998;36:243247.

5 Congpuong K, Sirtichaisinthop J, Tippawangkosol P, Suprakrob K, Nabangchang K, Tan-Ariya $\mathrm{P}$, Karbwang J: Incidence of antimalarial pretreatment and drug sensitivity in vitro in multidrug-resistant $P$. falciparum infection in Thailand Trans R Soc Trop Med Hyg 1998; 92:84-86.
6 Dar FK, Bayoumi R, AlKarmi T, Shalabi A, Beidas F, Hussein MM: Status of imported malaria in a control zone of the United Arab Emirates bordering an area of unstable malaria. Trans R Soc Trop Med Hyg 1993;87:617-619.

7 Malik G, Ghan A, Abdalla S: No response to chloroquine therapy in a case of a Saudi falciparum malaria. Saudi Med J 1997;18:99-100.

8 Kinsara AJ, Abdelaal MA, Jeje OM, Osoba AO: Chloroquine-resistant $P$. falciparum malaria: Report of two locally acquired infections in Saudi Arabia. Am J Trop Med Hyg 1997; 56:573-575.

9 Kinsara J: Chloroquine resistant $P$. falciparum malaria in Saudi patients. Saudi Med J 1997; 18:610.

10 Alrajhi A, Dhahab S, Alhazmi M: An outbreak of chloroquine resistant $P$. falciparum cerebral malaria in a chloroquine susceptible (abstract). Clin Infect Dis 1998;27:925.

11 WHO: In vitro micro-test (mark II) for the assessment of the response of $P$. falciparum to chloroquine, mefloquine, quinine, sulfadoxine/pyrimethamine and amodiaquine. Mimeographic document MAP/87.2, Revision 1. Geneva, World Health Organization, 1990
12 Anonymous: Probable locally acquired mosquito-transmitted Plasmodium vivax infection - Georgia, 1996. MMWR Morb Mortal Wkly Rep 1997;46:264-267.

13 Wilton P: Malaria may be on move to 'tropical' Canada. Can Med Ass J 1998;158:160.

14 WHO: Malaria situation in the world. World Health Organ Wkly Epidemiol Rec 1997;36:269-276.

15 Sharma YD, Biswas S, Pillai CR, Ansari MA, Adak T, Devi CU: High prevalence of chloroquine resistant $P$. falciparum infection in Rajasthan epidemic. Acta Trop 1996;62:135141

16 Dua VK, Kar PK, Gupta NC, Kar I, Sharma VP: In-vivo and in-vitro sensitivity of $P$. falciparum to chloroquine in Chennai (Tamil Nadu), India. Indian J Malariol 1997;34: $1-7$. 